\title{
Uma plataforma para comunicação eficiente entre discentes e instituições de ensino
}

\author{
Daniela Araujo ${ }^{1}$, Artur Kronbauer ${ }^{1,2}$, Jorge Campos ${ }^{1}$ \\ ${ }^{1}$ Programa de Pós-Graduação em Sistemas e Computação (PPGCOMP) - Universidade \\ Salvador (UNIFACS) - 41.770-235 - Salvador - BA - Brasil \\ ${ }^{2}$ Departamento de Ciências Exatas e da Terra (DCET II) - Universidade do Estado da \\ Bahia (UNEB) - 48.100-000 - Alagoinhas - BA - Brasil
}

\section{daniela.araujo@unifacs.br, arturhk@gmail.com,jorgeapcampos@gmail.com}

\begin{abstract}
One of the main problems in education is the pedagogical follow up and analysis of the student experience in the classroom. The ideal would be a follow-up before, during and after each class, making it possible to obtain contextualized information of the students' expectations and the didactics used by the professor in each class. In this sense, this article presents a platform that allows the collection of data related to the experience that the students are having with the methodology adopted by the professor in real-time. The platform is based on the submission of questions to the students after each class. The students use their smartphones to answer the questions. In this way, those responsible for academic quality control can follow the students' perceptions regarding methodological actions during each class, contributing to possible adjustments when necessary.
\end{abstract}

Resumo. Um dos problemas na área da educação é o acompanhamento pedagógico adotado pelos professores e a análise da experiência dos alunos em sala de aula. O ideal seria um acompanhamento antes, durante e após cada aula, possibilitando a obtenção de informações contextualizadas a expectativa dos alunos e a didática utilizada pelo docente. Nesse sentido, este artigo apresenta como contribuição uma plataforma que possibilita realizar, em tempo real, a coleta de dados relacionados à experiência dos discentes com a metodologia adotada pelo professor. A plataforma tem como base a submissão de perguntas aos alunos nos seus smartphones e o armazenamento das respostas numa base de dados. Desta forma, os responsáveis pelo controle da qualidade acadêmica ou o próprio professor podem acompanhar a percepção dos alunos com relação às ações metodológicas no decorrer de cada aula e contribui para possíveis ajustes quando necessário.

\section{Introdução}

De acordo com o MEC, as Instituições de Ensino Superior (IES) devem utilizar ferramentas para medir a satisfação dos discentes com relação ao processo de ensinoaprendizagem que são gerenciados pela Comissão Própria de Avaliação (CPA) [MEC 2004]. Normalmente, este tipo de avaliação é realizado no formato de questionários 
VIII Congresso Brasileiro de Informática na Educação (CBIE 2019)

Anais do XXX Simpósio Brasileiro de Informática na Educação (SBIE 2019)

disponibilizados em páginas web, tornando o processo cansativo e desvinculado do que realmente aconteceu no dia-a-dia da sala de aula.

O convencimento dos discentes para se engajarem no processo de avaliação é outro ponto crucial. Muitas vezes, os alunos não apresentam motivação para responder as perguntas, levando as IES a obrigarem os discentes a participarem do processo, restringindo o acesso às funcionalidades do sistema acadêmico até que o aluno responda a todas as perguntas.

Outro ponto relacionado com o assunto em discussão é a evasão escolar. Segundo estudos de [Stoffel e Ziza 2014], apesar do baixo rendimento acadêmico não ser um motivo manifestado pelos alunos no momento de seu pedido de trancamento, há uma clara relação entre o baixo rendimento escolar e a evasão do curso. Os autores sugerem criar estratégias para estimular a satisfação dos alunos e identificar dificuldades de aprendizagem nos primeiros momentos em que forem sinalizados.

Neste contexto, o objetivo desta pesquisa é propor uma plataforma que possibilite a realização do processo avaliativo em tempo real, utilizando o próprio smartphone do discente, sendo esta uma tecnologia utilizada com frequência por eles.

Como contribuição, espera-se que os dados possam ser analisados por coordenadores, professores e responsáveis pela qualidade do processo de ensinoaprendizagem da instituição, possibilitando que os ajustes possam ser realizados de forma rápida e eficaz, em um curto espaço de tempo.

Na próxima seção, será descrita a técnica utilizada para a coleta de dados de acordo com a abordagem proposta. $\mathrm{Na}$ Seção 3, serão discutidos alguns trabalhos correlatos. A Seção 4 descreverá a concepção da plataforma. Na Seção 5, será apresentado um estudo de caso para verificar as potencialidades da plataforma. A Seção 6 descreve os resultados encontrados. Por fim, a Seção 7 relata as conclusões e futuros trabalho.

\section{Técnica para a coleta de dados no contexto acadêmico}

Apesar do termo User eXperience (UX) ter uma relação estreita com a área de Interação Homem-Computador, seu conceito amplo permite analisar como as pessoas se sentem ao interagir com um produto ou serviço, independentemente de existir relação com dispositivos computacionais [Lachner et al. 2016].

$\mathrm{Na}$ UX o indivíduo tem contato com o produto ou serviço dentro de um contexto, considerando-se que fatores externos modificam a experiência, além de haver intercorrência de influências sociais e culturais [Hassenzahl et al. 2015]. Outro ponto a ser ressaltado é a temporalidade da interação, que pode sofrer mudanças ao longo do tempo. [Norman 2004] defende que os mínimos detalhes contribuem para uma UX agradável, prazerosa e inesquecível, preservando e fidelizando os consumidores.

Neste sentido, boas experiências por parte dos discentes nas IES pode ser a chave para o sucesso do aprendizado. Entretanto, um dos principais desafios é conseguir abrir um canal de comunicação entre discentes e instituições que possibilite mensurar e separar as boas das más experiências. Para a especificação de um canal de comunicação moderno e eficiente, foram investigadas técnicas que permitissem coletar a UX através 
VIII Congresso Brasileiro de Informática na Educação (CBIE 2019)

Anais do XXX Simpósio Brasileiro de Informática na Educação (SBIE 2019)

de questionários de autorrelato, que fossem apropriadas para serem aplicadas na abordagem idealizada neste trabalho.

Apesar de existir várias técnicas de avaliação de UX [da Silva Júnior 2018], nem todas permitem que os usuários consigam expressar completamente os seus sentimentos [Nakamura et al. 2017]. Neste sentido, os principais requisitos que se buscou na escolha da técnica foram: a possibilidade de se realizar interlocuções em tempo real, de forma simples, rápida e utilizando o smartphone do discente. Sendo assim, foi escolhida a Experience Sampling Method (ESM) [Larson 1983].

Segundo [Larson 1983], a técnica ESM possibilita medir duas dimensões: o tipo de emoção (positiva ou negativa) e a intensidade da emoção. Para isso, é usado um conjunto de figuras indicando estados emocionais relacionados à pergunta. A sequência das figuras refere-se à intensidade da emoção e podem ser interpretadas da esquerda para a direita (Figura 1) como: muito satisfeito, satisfeito, indiferente, insatisfeito e muito insatisfeito.

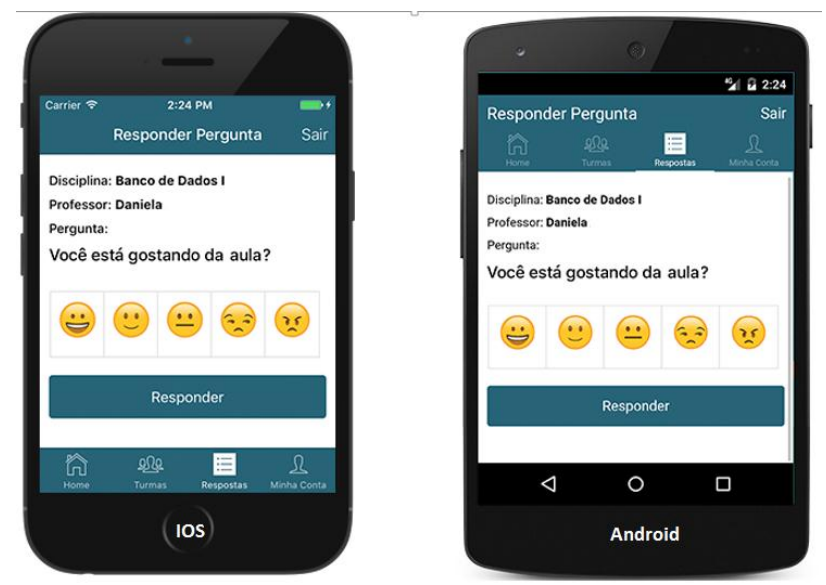

Figura 1. Aplicativo aSense (Academic Sense) utilizando a técnica ESM.

\section{Trabalhos Relacionados}

Atualmente, a utilização de técnicas de autorrelato para a coleta de dados referente à UX vem sendo explorada no meio coorporativo e científico. Entretanto, na maioria das avaliações observa-se que a interlocução com o público alvo ainda é por meio de questionários em papel ou em sites WEB. Desta forma, consideram-se apenas como trabalhos relacionados os que utilizaram a técnica ESM e foram implementados em dispositivos móveis com o objetivo de avaliar algum aspecto relacionado ao meio educacional. Neste contexto, foram selecionados quatro trabalhos:

- [Zirkel et al. 2015] avaliaram como os discentes, docentes e líderes escolares se envolvem com a educação e como esta interação influencia nas suas vidas pessoais.

- [Thomas e Azimitia 2016] realizaram um levantamento das experiências pedagógicas mediadas por tecnologias em sala de aula e a satisfação que estas trouxeram para os discentes e docentes.

- [Kronbauer et al. 2014] avaliaram a satisfação dos usuários com os aplicativos instalados em seus smartphones levando em consideração informações contextuais e 
VIII Congresso Brasileiro de Informática na Educação (CBIE 2019)

Anais do XXX Simpósio Brasileiro de Informática na Educação (SBIE 2019)

sociais. Dentre os resultados relatados na pesquisa, ressaltam-se a frequência e os tipos de aplicativos utilizados no contexto acadêmico.

- [Xie et al 2019] realizaram uma investigação com 133 estudantes de graduação e mediram o envolvimento afetivo, cognitivo e comportamental através de perguntas antes, durante e após a realização das aulas com o objetivo de encontrar padrões e diferenças de comportamento entre os estudantes, além de avaliar a sua relação com as disciplinas e professores.

A partir da análise referente a finalidades de cada pesquisa mencionada, é possível verificar que se destinam a diferentes fins, mesmo sendo aplicadas no contexto acadêmico. A proposta de [Xie et al 2019] é a que mais se aproxima dos objetivos para o qual a plataforma aSense, que será apresentada na próxima seção, se destina. Entretanto, é fundamental destacar que a plataforma aSense foi modelada e construída com funcionalidades específicas para o contexto acadêmico, contemplando módulos específicos para o cadastro e relacionamentos de turmas, professores, alunos e câmpus universitários. Além disso, contempla um módulo de visualização de resultados que permite que os responsáveis institucionais pela qualidade acadêmica possam analisar os dados coletados em tempo real.

\section{A Plataforma aSense}

A plataforma aSense (Academic Sense) foi concebida para remodelar a forma de comunicação entre discentes e a instituição de ensino, utilizando tecnologias modernas. As mensagens são programadas por professores ou administradores (coordenadores) com previsão de quando e para quem serão disparadas. Assim, podem ser medidas as expectativas e as motivações dos alunos em relação a metodologia do professor, infraestrutura e serviços oferecidos pela instituição de ensino.

Os requisitos funcionais podem ser relacionados como segue:

- Possibilitar cadastros de professores, disciplinas, turmas, atividades, perguntas e estabelecer cronograma de envio de enquetes aos alunos.

- Disponibilizar um aplicativo móvel para funcionar como interface de comunicação entre alunos e professores/administradores, possibilitando a recepção de perguntas e a transmissão das respostas dos alunos.

- Prover um Servidor Web para disparar perguntas programadas para os alunos e receber as respostas em um Sistema Gerenciador de Banco de Dados (SGBD).

- Disponibilizar ferramentas para analisar as respostas e identificar os resultados das enquetes realizadas.

A plataforma foi desenvolvida utilizando os conceitos previstos no ServiceOriented Architecture (SOA) [Erl 2018] e a forma de comunicação entre os componentes utiliza o Javascript Object Notation (JSON).

\subsection{Unidade de Cadastramento}

Esta unidade disponibiliza uma interface Web para o coordenador do curso (administrator) cadastrar: cursos, disciplinas, turmas, atividades, câmpus (unidades), 
VIII Congresso Brasileiro de Informática na Educação (CBIE 2019)

Anais do XXX Simpósio Brasileiro de Informática na Educação (SBIE 2019)

professores, convidar coordenadores (associar quem irá administrar a enquete), perguntas e agendamento das mensagens.

As perguntas são previamente agendadas pelo administrador do sistema (CPA ou coordenador de curso) e enviadas a uma turma específica, tendo como receptores todos os discentes vinculados a essa turma.

\subsection{Unidade de Transmissão e Coleta de Dados}

Esta unidade prevê a construção de um aplicativo móvel chamado de aSense (Figura 1). $\mathrm{O}$ aluno (cliente) precisa ter o aplicativo instalado em seu smartphone para responder a enquete e cadastrar os seus dados, tais como, curso e turma.

Para conceber o aplicativo para os sistemas operacionais Android e iOS foi adotado o modelo de desenvolvimento híbrido que permite a construção de aplicativos móveis utilizando-se de HTML, CSS e Javascript.

As funcionalidades do aplicativo aSense estão estruturadas em três serviços:

- Receber mensagens ou alertas no dispositivo sem a necessidade do aplicativo estar em execução.

- Coletar dados com uso da técnica ESM.

- Transferir os dados coletados para a Unidade de Armazenamento de Dados disponibilizado no Servidor Web.

\subsection{Unidade de Armazenamento de Dados}

Esta unidade utiliza um SGBD que permite a persistência dos dados, controle de redundâncias, segurança, suporte a transações, utilização de regras de restrição de integridade e independência de dados. O Servidor Web faz a intermediação dos dados na comunicação com a Unidade de Cadastramento e a Unidade de Transmissão e Coleta de Dados.

Os dados são armazenados no SGBD MySql Server, hospedado em nuvem no Google Cloud Platform e disponibiliza todos os dados para serem analisados pela Unidade de Correlação de Dados.

\subsection{Unidade de Correlação de Dados}

Esta unidade possibilita a análise dos dados pelos administradores e coordenadores. Ela deve utilizar os dados oriundos da unidade de Armazenamentos de Dados para gerar os gráficos e dashboards.

A Unidade de Correlação de Dados utiliza o Power BI, uma ferramenta de Business Intelligence (BI) [Sharda et al. 2014], para criação de dashboard com informações e gráficos dinâmicos e interligados.

\section{Experimento para avaliar a plataforma aSense}

A execução do experimento para avaliar a plataforma apresentada nesta pesquisa tomou como base a metodologia proposta pelo framework DECIDE [Sharp et al. 2011] que norteou a especificação dos passos realizados durante todas as fases do experimento. 
VIII Congresso Brasileiro de Informática na Educação (CBIE 2019)

Anais do XXX Simpósio Brasileiro de Informática na Educação (SBIE 2019)

\subsection{Determinar o objetivo da análise}

O foco do experimento foi obter informações sobre a eficácia e a eficiência da plataforma, bem como avaliar a experiência dos discentes, com um interesse especial em analisar a satisfação dos alunos antes, durante e após as aulas.

\subsection{Explorar perguntas a serem respondidas}

Tomando como base os objetivos a serem alcançados, foram elaboradas perguntas referentes a aspectos pedagógicos das disciplinas como, por exemplo:

- Você está motivado para a aula que terá hoje?

- Você está gostando da aula que está acontecendo agora?

- Você gostou da aula que acabou de ter?

- Qual o seu grau de satisfação com a metodologia aplicada nesta aula?

\subsection{Escolher o método de avaliação}

Para responder as questões definidas na subseção anterior, foram submetidas perguntas aos participantes utilizando a técnica ESM. As perguntas foram enviadas antes, durante e depois das aulas, com o objetivo de obter uma avaliação subjetiva a respeito da experiência do aluno com a metodologia aplicada pelo professor.

Para coletar a percepção dos discentes, foi utilizada a escala de [Likert 1932], onde as opções de respostas variaram de 1 a 5, sendo 1 - "Concordo Plenamente" e 5 "Discordo Plenamente".

\subsection{Identificar e administrar as questões práticas}

O experimento foi realizado com alunos dos cursos de Sistemas de Informação, Ciência da Computação e Engenharias de Computação de uma instituição com a participação de 9 turmas, num total de 5 disciplinas e 5 professores em 2 turnos (matutino e noturno). $\mathrm{O}$ experimento foi realizado com 147 alunos durante 4 meses.

Nesta fase, foram levantados inúmeros pré-requisitos, dentre os quais podem ser destacados: (i) a seleção das turmas; (ii) análise dos planos de aula dos professores; (iii) elaboração do cronograma de agendamento das perguntas em função do plano de aula dos docentes; (iv) apresentação do projeto para engajamento dos alunos; (v) disponibilização do aplicativo no Google Play (para Android) e na App Store (para iOS).

\subsection{Decidir como lidar com as questões éticas}

O projeto foi submetido ao Comitê de Ética para a devida avaliação e aprovado para execução com o Parecer Consubstanciado de número 1.973 .433 e Certificado de Apresentação para Apreciação Ética (CAAE) de número 64728316.0.0000.5033.

\subsection{Estabelecer formas de avaliar, interpretar e apresentar os resultados}

A apresentação dos resultados foi realizada em forma de gráficos com dados quantitativos referentes à Avaliação Pedagógica e usando estatística descritiva. Para preservar o anonimato dos participantes os cursos foram denominados de Curso A, B e C; as disciplinas de Disciplina 1, 2, 3, 4 e 5 e os professores de Professor A, B, C, D e E. 
VIII Congresso Brasileiro de Informática na Educação (CBIE 2019)

Anais do XXX Simpósio Brasileiro de Informática na Educação (SBIE 2019)

\section{Resultados e análise da execução do experimento}

Devido ao limite de páginas desse artigo, serão apresentadas, nesta seção, alguns dos resultados obtidos no experimento que servem para exemplificar as potencialidades da plataforma aSense, entretanto, inúmeras outras constatações são factíveis de se realizar.

A pesquisa da satisfação dos alunos na aula pode sofrer variações nos índices conforme as métricas selecionadas. O gráfico interativo da Figura 2 mostra que é possível filtrar o curso, a disciplina, a turma, o professor, a atividade, o sexo, se trabalha e se possui carro, além de permitir a combinação desses filtros. O resultado apresentado na Figura 2 abrange toda a amostra de participantes, totalizando 147 discentes, sendo observado que $64,7 \%$ dos discentes estão satisfeitos ou muitos satisfeitos, $19,86 \%$ são indiferentes e $15,44 \%$ estão insatisfeitos ou muito insatisfeitos.

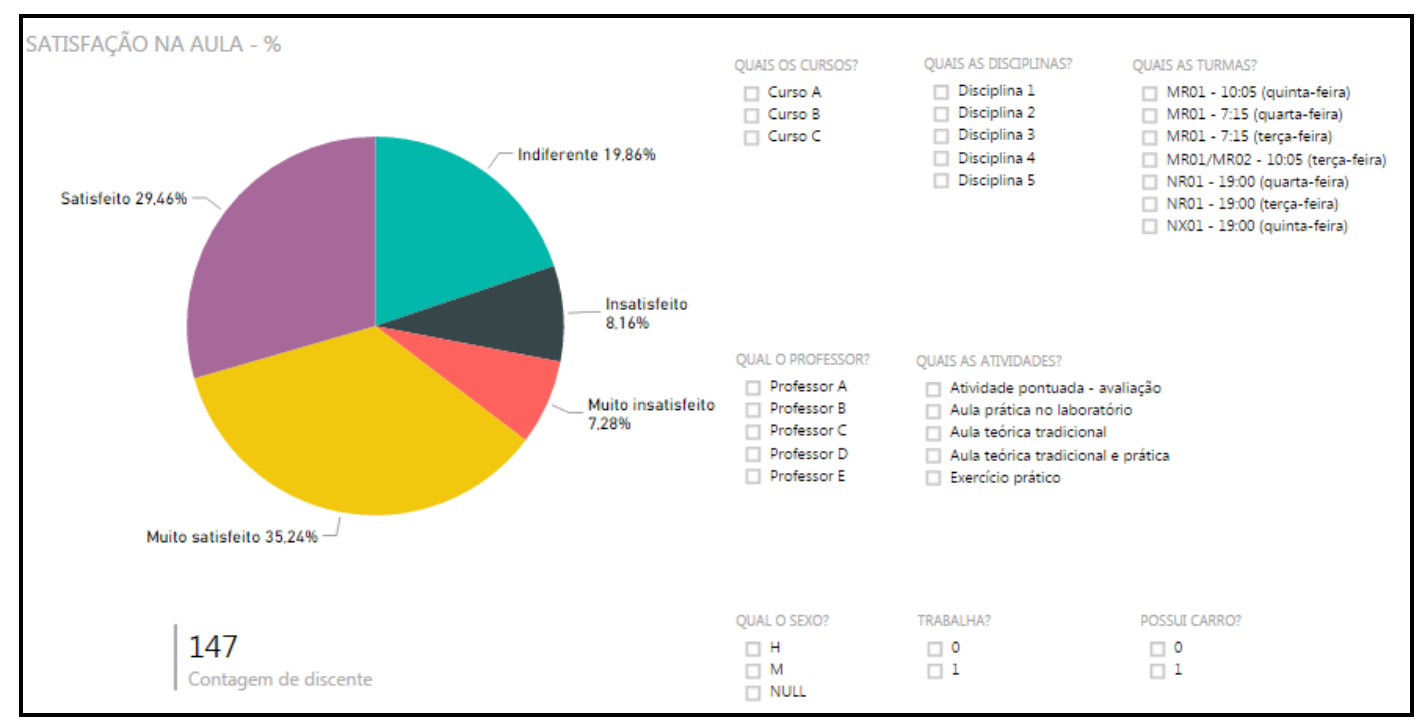

Figura 2. Gráfico interativo da satisfação na aula.

A Figura 3 representa o dashboard das atividades realizadas nas aulas e o efeito na satisfação associado às disciplinas e aos professores. Nele são apresentados o percentual total de atividades desenvolvidas nas aulas durante o experimento (Gráfico A), as médias das motivações dos alunos por disciplina (Gráfico B), por professor (Gráfico D) e o percentual de atividades realizadas por cada professor (Gráfico C). As disciplinas 1 e 4 são as mais bem avaliadas com média de 4,23 e 3,94 respectivamente (Gráfico B). Os professores A e D obtiveram as melhores médias com 4,23 e 4,04 (Gráfico D).

Os professores melhor avaliados utilizaram estratégias pedagógicas diferentes. $\mathrm{O}$ Professor A utilizou 79,28\% do tempo com Aulas Teóricas Tradicionais e Exercícios Práticos, já o Professor D utilizou 90,1\% do tempo com Aulas Práticas no Laboratório e Aulas Teóricas Tradicionais e Práticas conforme pode ser observado na Figura 3 Gráfico C.

O dashboard da satisfação na aula ilustrado na Figura 4 - Gráfico A mostra que a média da satisfação dos alunos participantes do experimento melhora ao longo das aulas. Em linhas gerais, a média da satisfação antes da aula é de 3,48, durante a aula passa para 3,91 e ao final da aula atinge 3,95. Esse resultado é um indício de que as 
VIII Congresso Brasileiro de Informática na Educação (CBIE 2019)

Anais do XXX Simpósio Brasileiro de Informática na Educação (SBIE 2019)

aulas foram bem ministradas, pois os alunos chegam relativamente motivados e se mantêm com satisfação crescente até o final, apesar de se perceber pelos resultados que existe possibilidade de melhorar a satisfação.

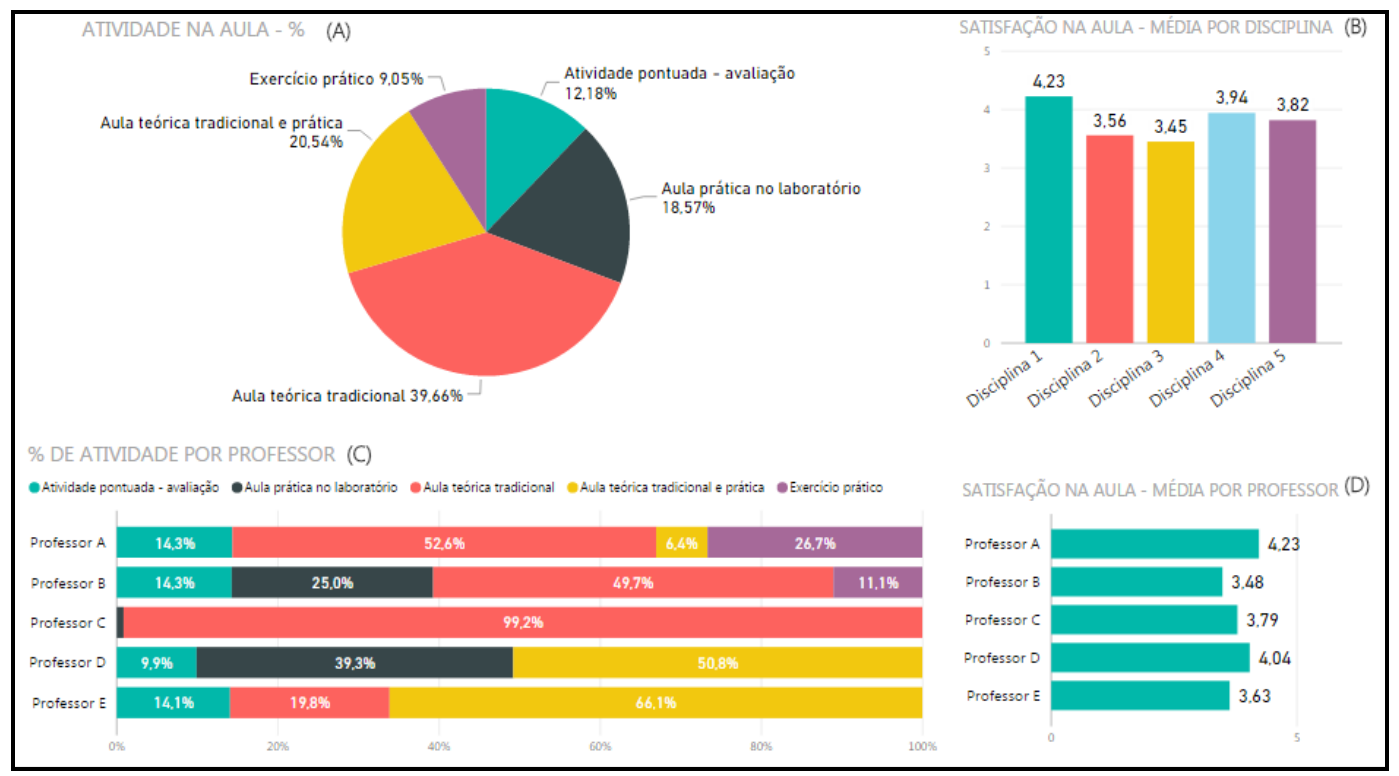

Figura 3. Dashboard das atividades e efeitos na satisfação associados à disciplina e professor.

Ainda no dashboard da Figura 4 - Gráfico B observa-se que 64,6\% consideram-se satisfeitos ou muito satisfeitos e 19,92\% indiferentes. A distribuição das respostas por curso é de $33,63 \%$ para o Curso A, $25,72 \%$ para o Curso B e $40,65 \%$ para o Curso C, conforme o Gráfico C.

Utilizando o Power BI é possível fazer inúmeros tipos de filtros, como identificar em quais cursos os alunos entram mais motivados e o comportamento ao longo de um semestre ou, ainda, em quais cursos as aulas têm um poder motivacional maior.

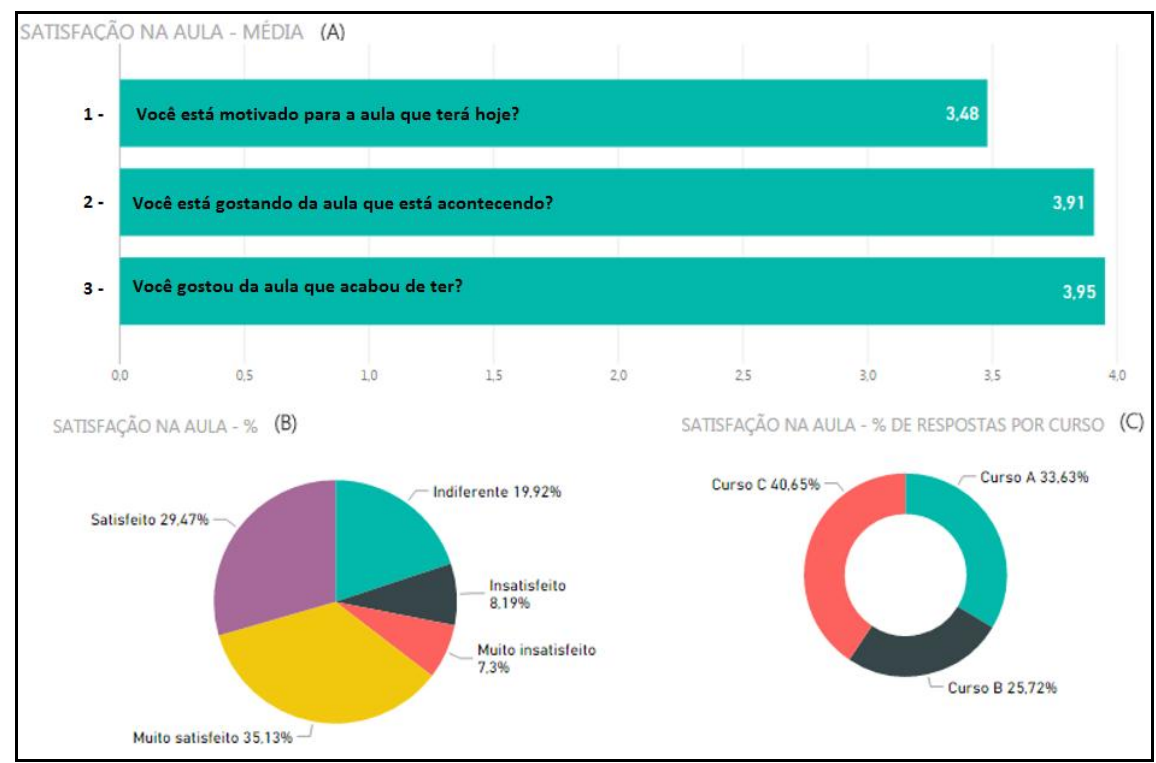

Figura 4. Dashboard da satisfação na aula. 
VIII Congresso Brasileiro de Informática na Educação (CBIE 2019)

Anais do XXX Simpósio Brasileiro de Informática na Educação (SBIE 2019)

\section{Conclusões e trabalhos futuros}

A presente pesquisa teve por objetivo criar uma plataforma para abrir um canal de comunicação entre discentes e a IES, permitir a captura da experiência dos alunos em relação ao conteúdo e metodologia empregados nas aulas e possibilitar a realização de análises dinâmicas dos resultados.

No desfecho deste estudo conclui-se que a aplicação de técnicas de medição da UX quando praticadas dentro do contexto educacional e com uso de tecnologias modernas revelam resultados ricos para decisões dos gestores acadêmicos, além de propiciar aos professores a possibilidade de aprimorar as suas práticas metodológicas.

A plataforma com o uso do Power BI traz uma riqueza de variações para as análises. À medida que as variáveis são manipuladas, descobertas de conhecimento surgem para que os negócios e as decisões sejam embasados em evidências. Um coordenador de curso tem, a partir das visões disponibilizadas pela plataforma, condições de agir de forma diferente e adequada para cada tipo de situação conforme a análise dos resultados.

Vale ressaltar que as conclusões obtidas no experimento não são passíveis de generalização, pois sendo aplicados em um contexto com variantes diferentes, os resultados provavelmente não serão os mesmos. O experimento serviu para testar a plataforma e revelar as potencialidades da ferramenta para prover diversificadas formas de avaliação.

Como futuros trabalhos, seria interessante realizar estudos entre alunos de cursos com menor familiaridade com as tecnologias e uma análise por um tempo maior para observar se haverá redução de uso ou queda na satisfação com a plataforma. Além disso, seria conveniente avaliar o impacto da utilização da ferramenta no decorrer das aulas e o quanto pode interferir na atenção dos alunos.

Um ponto importante é criar uma unidade para o docente na plataforma. Assim, os professores podem registrar as atividades com mais detalhes, cancelar aula, programar aula extra e acompanhar os resultados. Estudos sobre a correlação entre a satisfação do aluno nas aulas, o seu desempenho acadêmico e a evasão são de suma importância e podem ser tratados em trabalhos futuros.

A utilização da plataforma aSense pode representar uma contribuição para medir e analisar a satisfação dos universitários brasileiros. Pode ser um importante passo para intervenções no ambiente educacional visando promover a satisfação, colaborar com a diminuição da evasão escolar e contribuir com o processo didático-pedagógico.

\section{Referências}

Erl, T. (2008). “SOA: principles of service design”, Upper Saddle River: Prentice Hall.

Hassenzahl, M., Wiklund-Engblom, A., Bengs, A., Hägglund, S. and Diefenbach, S. (2015). "Experience-oriented and product-oriented evaluation: psychological need fulfillment, positive affect, and product perception", International journal of humancomputer interaction 31(8): 530-544. 
VIII Congresso Brasileiro de Informática na Educação (CBIE 2019)

Anais do XXX Simpósio Brasileiro de Informática na Educação (SBIE 2019)

Kronbauer, A. H., Machado, D. and Santos, C. A. (2015). "Capture and analysis of interaction data for the evaluation of user experience with mobile devices". Int. Conference of Design, User Experience, and Usability. Springer, Cham, 54-65.

da Silva Júnior, J. B. and Kronbauer, A. H. (2018). "A Study of Hedonic Experience Related to UX Capture Techniques". In Proceedings of the 17th Brazilian Symposium on Human Factors in Computing Systems, ACM, 4.

Lachner, F., Naegelein, P., Kowalski, R., Spann, M. and Butz A. (2016). "Quantified UX: Towards a Common Organizational Understanding of User Experience", proc. of the $9^{\text {th }}$ Nordic Conference on Human-Computer Interaction. ACM, 56.

Larson, R., Csikszentmihalyi, M. (2014) “The Experience Sampling Method”, in Flow and the Foundations of Positive Psychology. Springer, Dordrecht, 21-34.

Likert R. (1932). “A Technique for the Measurement of Attitudes". Archives of Psychology, 140, 1-55.

MEC. (2004). "Regulamenta os procedimentos de avaliação do Sistema Nacional de Avaliação da Educação Superior (SINAES)", instituído na Lei no 10.861, de 14 de abril de 2004. Portaria No 2.051.

Nakamura, W. T., Marques, L. C., Rivero, L., de Oliveira, E. H. T. and Conte, T. (2017). "Are Generic UX Evaluation Techniques Enough? A study on the UX Evaluation of the Edmodo Learning Management System", Brazilian Symposium on Computers in Education, 28(1): 1007-1016.

Norman, D. A. and Nielsen, J. (2016). "The Definition of User Experience", https://www.nngroup.com/articles/definition-user-experience/

Thomas V., Azmitia M. (2016). "Tapping Into the App: Updating the Experience Sampling Method for the $21^{\text {st }}$ Century". Emerging Adulthood 4(1): 60-67.

Sharda, R., Delen D. and Turban, E. (2014). "Business Inteligence and Analytics: Systems for Decision Support". $10^{\text {th }}$ Ed. Pearson.

Sharp, H., Rogers, Y. and Preece, J. (2011). "Interaction design: beyond humancomputer interaction". $3^{\text {rd }}$ ed. New York: John Wiley \& Sons.

Stoffel, W. P. and Ziza, C. R. (2014). "Evasão escolar em cursos superiores: Estudo comparativo entre os pedidos de trancamento e o aproveitamento escolar". IX Simpósio Pedagógico e Pesquisas em Comunicação.

Xie, K.; Heddy, B. C. and GREENE, B. A. (2019). "Affordances of using mobile technology to support experience-sampling method in examining college students' engagement”. Computers \& Education, v. 128, p. 183-198, 2019.

Zirkel, S., Garcia, J. A. and Murphy, M. C. (2015). "Experience-Sampling Research Methods and Their Potential for Education Research". Educational Researcher 44(1): 7-16. 